\title{
Control of the conversion between circularly polarized photons and spin by introducing chiral orbit in organic complexes
}

Renjie $\mathrm{Hu}^{1}$ and Wei Qin (1) ${ }^{1}$

\begin{abstract}
Spin tunability by circularly polarized photons occurs in an unconnected method, providing more spaces to design memory devices. Here, by involving an artificially fabricated organic chiral structure, we let circularly polarized photons interact with the electron spins of organic materials. The spiral potential field of the chiral structure can endow electrons with additional orbital angular momentum to realize coupling between circularly polarized photons and electron spins. Switching the incident light from linearly to circularly polarized light, while keeping the intensity constant, results in the magnetic field-decreased photoluminescence changing to magnetic field-enhanced photoluminescence. Because the interaction between right-handed circularly polarized photons and chiral lattices is stronger in the fabricated chiral complexes, tuning photoluminescence by a magnetic field is more pronounced under the stimulus of right-handed circularly polarized light. In addition, applying an external bias and changing the temperature of organic chiral complexes are tested to further study the conversion between circularly polarized photons and spins. It is expected that this study could help us to further understand the coupling between spins and circularly polarized photons in organic chiral materials.
\end{abstract}

\section{Introduction}

As electrons couple to electromagnetic fields not only via charge but also via spin degrees of freedom ${ }^{1,2}$. The interaction between electron spins and the electromagnetic fields that generate large magneto-optical coupling effects has been observed in many inorganic materials $s^{3-5}$. One of the most widespread methods to tune spin is the optical orientation of electron spins by circularly polarized light in inorganic materials, with the orbital angular momentum acting as an intermediate $^{6-9}$. However, in pure organic materials, it is challenging to control the spin by applying circularly polarized light. Thus, extending the coupling between spin and circularly polarized photons from inorganic materials to organic materials should be an interesting and fascinating research area, which will lead to ground-breaking development in the area of organic spintronics.

\footnotetext{
Correspondence: Wei Qin (wqin@sdu.edu.cn)

${ }^{1}$ School of Physics, State Key Laboratory of Crystal Materials, Shandong

University, 250100 Jinan, China
}

In organic materials, electrons generated by excitation or external injection are no longer extended states but are coupled with the crystal lattice and consequently form polarons or bipolarons with abundant charge-spin relationships ${ }^{10-15}$. The localized electronic states caused by strong electron-phonon coupling may lead to spontaneous electron spin polarization in well-designed organic structures without any magnetic element doping $^{16-20}$. By applying an external bias or changing the interchain coupling strength ${ }^{21-28}$, electron-phonon coupling will be changed to affect the localization of electrons, where the overall spin polarization is changed. However, because of the extremely weak orbital angular momentum in pure organic materials ${ }^{29}$, circularly polarized light cannot affect the orbital angular momentum to change the localization to further tune the spin polarization. Thus, it is very difficult to transfer the angular momentum of photons to that of the electron spins in pure organic materials. 
The chirality-induced spin selectivity (CISS) effect is a very important phenomenon in chiral structures, which means that one spiral structure only allows carriers with a specific spin direction to pass through. The CISS effect provides a unique way to generate local magnetization ${ }^{30-32}$, change the fluorescence time ${ }^{33}$, and photoluminescence ${ }^{34}$ on one side of the chiral molecules. The CISS-related effects are mainly based on ordered spiral structures to maximize the spin polarization.

Here, by involving artificially fabricated organic chiral structures randomly distributed on a substrate, we attempt to let the circularly polarized photon interact with the electron spin in organic materials, where the interaction among the photon, phonon, and electron spin is explored. The inherent spiral potential field of the chiral structure can endow the electrons with additional orbital angular momentum to realize the coupling between the photon and electron spin. Moreover, linearly and righthanded and left-handed circularly polarized light with identical intensity can be used to excite organic chiral materials to understand the conversion of angular momentum. Furthermore, the external bias dependence of electron localization acts as an effective way to understand the conversion. In addition, we also consider the temperature dependence of charge localization on the interaction between electron spins and circularly polarized photons.

\section{Results and discussion}

In achiral materials, a large density of electron-hole $(\mathrm{e}-\mathrm{h})$ pairs will be generated under light illumination, where the magnetic field could increase the ratio of triplet states to decrease the strength of fluorescence. As a result, M-PL (magnetic field dependence of photoluminescence) is negative for an achiral structure (the inset of Fig. 1a), where $\mathrm{M}-\mathrm{PL}$ is $(\mathrm{PL}(\mathrm{B})-\mathrm{PL}) / \mathrm{PL}$. Even when switching the incident light from circularly to linearly polarized light, the signal of M-PL is still negative, indicating that the angular momentum of circularly polarized photons does not affect the spin of electrons in achiral materials. However, if chiral nanowire polythiophene (nw-PT) is induced, the case becomes different and interesting. Chiral nw-PTs were characterized via CD spectroscopy (Fig. 1b). CD spectroscopy of the sample can still be


Fig. 1 Basic properties of the chiral charge transfer complexes. a Magnetic field-dependent PL of the chiral nw-PT:PCBM complexes under identical intensities of linearly and circularly polarized light. The inset is the M-PL of achiral complexes under linearly and circularly polarized light. b CD spectra of the chiral nw-PT structure, achiral nw-PT structure, and chiral nw-PT:PCBM charge transfer complexes. The inset is the schematic diagram of the chiral nw-PT:PCBM charge transfer complexes. c Magnetic field-dependent dielectric constant of chiral nw-PT:PCBM films under identical intensities of linearly and circularly polarized light. The inset shows the architecture of the nw-PT charge transfer complex-based device. Without a magnetic field, linearly (d) and circularly (e) polarized light induced weakly and strongly localized states, respectively. Under the stimulus of a magnetic field, the localization became more pronounced under linearly $(\mathbf{f})$ and circularly $(\mathbf{g})$ polarized light, respectively. 
guaranteed when the acceptor PCBM is added, indicating that the addition of the acceptor does not damage the helix structure.

As shown in Fig. 1a, under the illumination of linearly polarized light, the M-PL of chiral nw-PT is negative (similar to the achiral structure). However, switching the incident light from linearly to circularly polarized light could induce the M-PL to change from negative to positive in chiral nwPT. It is expected that there is a conversion between spin and circularly polarized photons in organic chiral structures. In addition to M-PL, the magnetic field dependence of the dielectric constant $(\mathrm{M}-\varepsilon)$ in chiral nw-PTs also shows pronounced differences when changing the incident light from linearly to circularly polarized light (Fig. 1c) with identical intensity (Fig. S1b, Supporting Information). Moreover, switching the incident light from right-handed to left-handed circularly polarized light could further tune the magnitudes of both M-PL and M- $\varepsilon$ (Fig. 1a, c). In the following, we will study the mechanism behind the above discussed phenomena in chiral structures.

Under light illumination, $\mathrm{e}-\mathrm{h}$ pairs will be generated in nw-PT. Some of them will stay in nw-PT, and the other part will form charge transfer states and then dissociate between nw-PT and PCBM. Because of the larger electron-phonon coupling for $\mathrm{PCBM}^{35,36}$, the magnitude of electron spin polarization in PCBM is larger than that of the hole in nwPT. A net spin polarization can be formed in PCBM. Thus, light illumination could generate $\mathrm{e}-\mathrm{h}$ pairs in both nw-PT and the spin-polarized electrons in PCBM during these chiral charge transfer complexes (Fig. 1d). In chiral structures, chirality-generated orbital angular momentum can act as a good medium to realize coupling between circularly polarized photons and $\operatorname{spin}^{37-40}$, where circularly polarized light could enhance spin polarization. Thus, in the fabricated chiral nw-PT:PCBM charge transfer complexes, circularly polarized light could effectively enhance the spin polarization. Because the magnitude of spin polarization is highly dependent on the charge localization ${ }^{41}$, circularly polarized light-induced larger spin polarization will enhance the localization of charge in PCBM (Fig. 1e). As a result, the interaction between the polarized spin in PCBM and the dipole (e-h pair) in nw-PT decreases when switching the incident light from linearly (Fig. 1d) to circularly (Fig. 1e) polarized light in the chiral nw-PT:PCBM charge transfer complexes.

Applying an external magnetic field to chiral charge transfer complexes, two direct effects can be observed: (i) increasing the spin polarization to further enhance charge localization in PCBM and (ii) increasing the ratio of triplet $\mathrm{e}-\mathrm{h}$ pairs in nw-PT. Under the illumination of linearly polarized light, the interaction between spin (in PCBM) and $\mathrm{e}-\mathrm{h}$ (in nw-PT) is stronger, which leads to a larger distance (smaller exchange interaction) between the electrons and holes of $\mathrm{e}-\mathrm{h}$ pairs. As a result, a magnetic field could effectively increase the ratio of triplet $\mathrm{e}-\mathrm{h}$ pairs due to the smaller exchange interaction. Because singlet $\mathrm{e}-\mathrm{h}$ pairs mainly contribute to the PL, M-PL is negative under the illumination of linearly polarized light in chiral complexes.

Under circularly polarized light illumination, the charge in PCBM is strongly localized, which results in a very weak interaction between spin and $\mathrm{e}-\mathrm{h}$ in chiral complexes, where the distance between electrons and holes in e-h pairs decreases (very large exchange interaction). Therefore, a large exchange interaction will prevent an external magnetic field from tuning the singlet/triplet ratio. However, the magnetic field could enhance the localization to further weaken the interaction between spin and $\mathrm{e}-\mathrm{h}$ (Fig. 1g), where the distance between an electron and a hole decreases to increase the recombination of fluorescence. Thus, under the illumination of circularly polarized light on chiral complexes, a magnetic field could effectively increase the strength of PL, where effect $i$ is dominant, leading to a positive M-PL; this is completely different from the case under linearly polarized light illumination (Fig. 1a). In addition, if there is no chiral orbit (achiral complex) acting as an intermediate to lead to the coupling between circularly polarized photons and spins, circularly polarized light cannot change the localization in PCBM. As a result, M-PLs show the same response for linearly and circularly polarized light in achiral charge transfer complexes (the inset of Fig. 1a; Fig. S2, Supporting Information). Moreover, it should be noted that for the CISS, when only linear polarization was used, the same ratio of both types of spins were excited, and therefore, the spinrelated effects were smaller ${ }^{32}$. However, in this work, different phenomena in chiral charge transfer complexes are observed in which M-PL changes from positive to negative when switching incident light from circularly polarized light to linearly polarized light (Fig. 1a).

To further understand the coupling between circularly polarized photons and spin, an external bias is applied to tune M-PL in chiral charge transfer complexes. The turn-on voltage of the chiral charge transfer complex-based device is $0.4 \mathrm{~V}$, which means that if the applied bias voltage is greater than $0.4 \mathrm{~V}$, it may cause the injection of external carriers. To avoid charge injection, the applied bias is lower than the turn-on voltage of chiral nw-PT:PCBM-based devices (the inset of Fig. 2a). When an external bias is applied, the localized electron is partially decoupled from the high frequency phonon (phonons in optical mode) to reduce the overall localization (spin polarization), where the interaction between spin and dipole becomes stronger to enlarge the distance between electron and hole for e-h pairs (Fig. 2b). As a result, the magnetic field could more effectively increase the ratio of triplet states to result in a more apparent M-PL. As shown in Fig. 2a, M-PL becomes more pronounced with increasing applied bias under the stimulus of linearly polarized light (red line). For the external bias dependence of M-PL under circularly polarized light (Fig. 2a, blue and black 


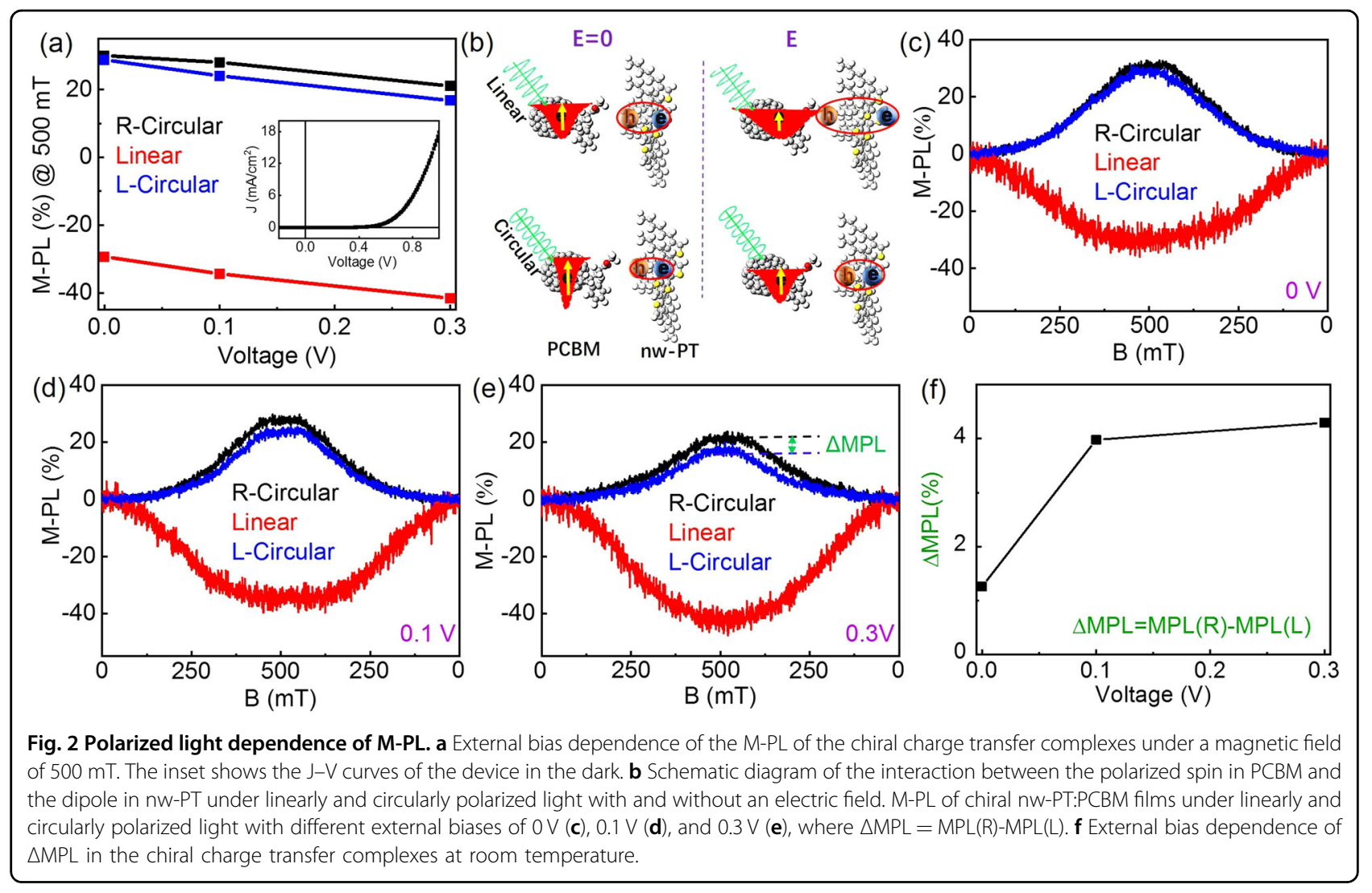

lines), the applied external bias will reduce the magnetic field-enhanced localization. Thus, the magnetic field cannot effectively tune the distance between electrons and holes for e-h pairs. As a result, M-PL decreases with increasing applied bias in chiral nw-PT:PCBM complexes under the illumination of circularly polarized light (Fig. 2a).

When switching incident light from right-handed to left-handed circularly polarized light with identical intensity, M-PL also shows a difference in chiral charge transfer complexes, $\triangle \mathrm{MPL}=\mathrm{MPL}(\mathrm{R})-\mathrm{MPL}(\mathrm{L})$, as shown in Fig. 2c-e and Figs. S3-6 (Supporting Information). Right-handed circularly polarized light results in better tunability of M-PL. It is known that fabricated chiral materials interact differently with left-handed and righthanded circularly polarized light, giving rise to circular dichroism. In these chiral nw-PT:PCBM charge transfer complexes, the CD signal is negative (Fig. 1b), indicating that the interaction between right-handed circularly polarized photons and the chiral lattice (localization) is stronger than that in the case of left-handed circularly polarized light. As a result, compared with left-handed circularly polarized light, right-handed circularly polarized light with its stronger photon-spin interactions could more effectively tune the localization to present a pronounced M-PL. Moreover, $\triangle \mathrm{MPL}$ increases with increasing applied bias in chiral complexes (Fig. 2f).
As discussed above, external bias could decrease the localization to enhance the spin-dipole interaction in chiral nw-PT:PCBM complexes. Dipoles in the complexes determine the magnitude of the dielectric constant. Applying an external bias to the devices, the dielectric properties will also be tuned. With strong spin-dipole interactions, the blocked dipoles cannot be more easily aligned by an external electric field. As shown in Fig. 3a, the dielectric constant decreases with increasing external bias. In addition, it should be noted that external bias cannot change the intensity of PL (Fig. 3b; Fig. S7, Supporting Information). This means that external bias cannot enlarge the distance between the electrons and holes in $\mathrm{e}-\mathrm{h}$ pairs. Thus, the external bias dependence of the $\mathrm{M}$ $\varepsilon$ change (Fig. $3 \mathrm{~d}$ ) is not caused by the change in the spin exchange interaction.

Under the stimulus of a magnetic field, both the ratio of triplet $\mathrm{e}-\mathrm{h}$ pairs and the electron localization will be enhanced in chiral nw-PT:PCBM complexes under linearly polarized light illumination. Because of the smaller dipolar moment for triplet $\mathrm{e}-\mathrm{h}$ pairs ${ }^{42,43}$, an increased magnetic field triplet $\mathrm{e}-\mathrm{h}$ pair ratio will decrease the magnitude of the dielectric constant. Moreover, magnetic field-enhanced localization of electrons will decrease the interaction between spin and $\mathrm{e}-\mathrm{h}$ to increase recombination, where the density of dipoles ( $\mathrm{e}-\mathrm{h}$ pairs) decreases 

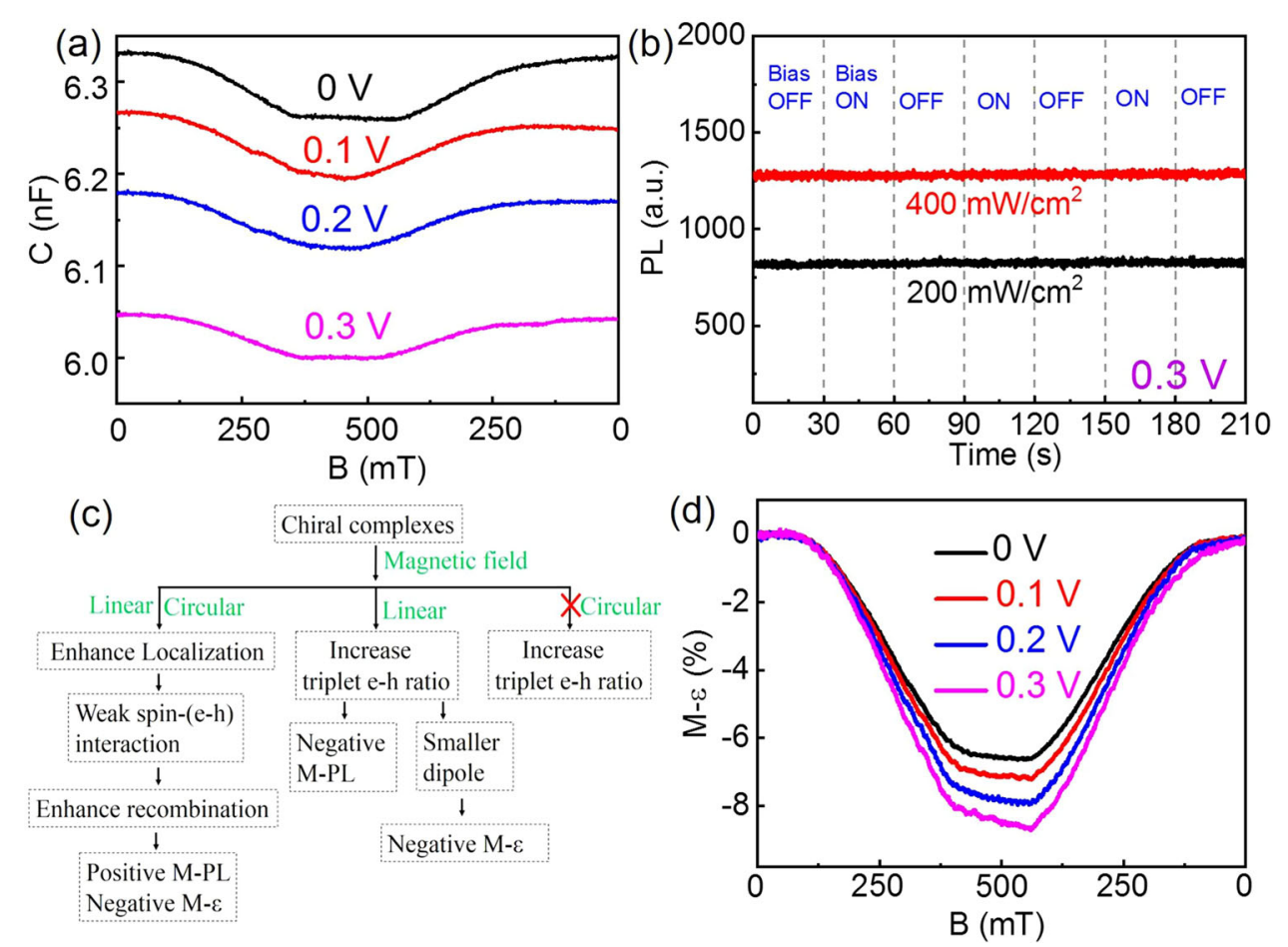

Fig. 3 Mechanism of the magnetic field dependence of properties. a Magnetic field-dependent capacitance of the chiral nw-PT:PCBM films with different external biases. $\mathbf{b}$ Dependence of bias on PL. ON (OFF) means the external bias is turned on (off). $\mathbf{c}$ Diagram of the magnetic field dependence of the dielectric constant. $\mathbf{d}$ M- $\varepsilon$ of the chiral nw-PT:PCBM charge transfer complexes with different external biases under linearly polarized light.

to further weaken the dielectric effect (Fig. 3c). As a result, $\mathrm{M}-\varepsilon$ is negative in chiral nw-PT:PCBM complexes under linearly polarized light illumination (Figs. 1c and 3d). However, under the stimulus of circularly polarized light, the channel of tunability of the singlet/triplet ratio by an external magnetic field will be blocked, where a weaker $\mathrm{M}-\varepsilon$ is observed compared with that under linearly polarized light with identical intensity (Fig. 1c and Figs. S8 and 9, Supporting Information).

In addition to the tunability of charge localization by external bias, the temperature change could also tune the localization to change the conversion between circularly polarized photons and spins. As shown in Fig. 4a, under circularly polarized light illumination, M-PL is strongly dependent on the temperature. M-PL increases with decreasing temperature and then decreases. As shown in Fig. $4 \mathrm{~b}$, increasing the temperature can generate two effects in chiral charge transfer complexes: (i) decreasing the PL intensity and (ii) decreasing the spin polarization (magnetic moment). A stronger PL at low temperature indicates that more $\mathrm{e}-\mathrm{h}$ pairs exist in the chiral complexes, where an external magnetic field could more effectively tune the singlet/triplet ratio to present a pronounced M-PL (from 300 to $150 \mathrm{~K}$ ). However, it should be noted that decreasing the temperature could also enhance localization to increase the magnitude of the magnetic momentum (spin polarization) in chiral charge transfer complexes (Fig. 4b). Low temperature-induced strong spin polarization will prevent an external magnetic field from tuning the charge localization. As a result, the magnetic field has little effect on the tunability in the interaction between spin and $\mathrm{e}-\mathrm{h}$ pairs when the temperature is decreasing. Thus, a threshold of temperature dependence of M-PL will be generated with decreasing temperature in chiral charge transfer complexes. It should be noted that for the CISS dependence of the localization $^{44}$, as shown in Fig. S10a, circularly polarized lightgenerated polarized spins in quantum dots will be transported (or blocked) due to the CISS effect to present spin delocalization (or localization). Here, by applying an external field (magnetic field or electric field), electron-phonon coupling in organic materials will be tuned to affect localization (Fig. S10b), where spin polarization is changed. Notably, the dependance of electron-phonon coupling on localization is independent of charge transport.

\section{Conclusion}

In summary, we fabricated organic chiral charge transfer complexes by spin-coating to study the conversion 

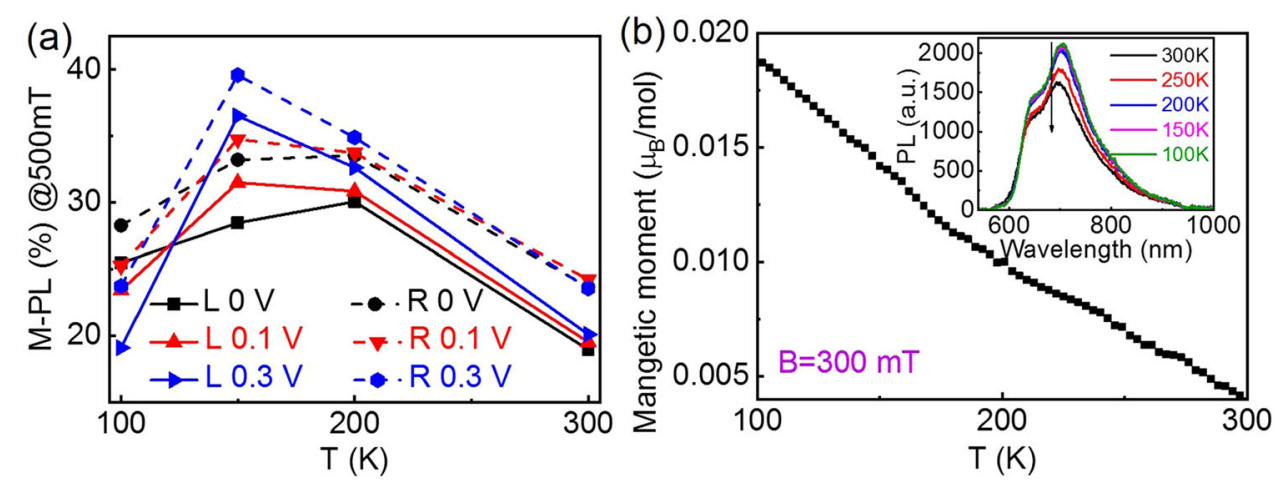

Fig. 4 Temperature dependence of properties in the charge transfer complexes. a Temperature-dependent M-PL under a magnetic field of 500 $\mathrm{mT}$ with different external biases. The solid line indicates left-handed circularly polarized light, and the dashed line indicates right-handed circularly polarized light. $\mathbf{b}$ Temperature dependence of the spin polarization of chiral nw-PT:PCBM charge transfer complexes. The inset shows the PL of the chiral nw-PT:PCBM charge transfer complexes at different temperatures.

between circularly polarized photons and spin through photon-orbit-spin interactions, thereby avoiding the intervention of the CISS effect. The inherent spiral potential field of the chiral structure can endow the electrons with additional orbital angular momentum to realize the coupling between circularly polarized photons and electron spins. Applying circularly polarized light to chiral charge transfer complexes, charge localization can be tuned through photon-orbit-spin coupling. Switching the incident light from linearly to circularly polarized light, M-PL will change from negative to positive in organic chiral complexes. Because the interaction between right-handed circularly polarized photons and chiral lattices is stronger in the fabricated chiral complexes, M-PL is more pronounced under the stimulus of right-handed circularly polarized light. In addition, we explored the influence of bias and temperature on the magnetic field effect with different polarized light excitations. Both the bias and temperature could change the charge localization in PCBM to further affect the conversion between photons and spins. Hopefully, this work can promote the understanding of photon-spin couplings and the better application of organic chiral materials in the area of organic spintronics.

\section{Experimental method}

\section{Sample preparation}

To prepare the organic chiral charge transfer complexes, PCBM and polythiophene were purchased from Sigma Aldrich (Shanghai, China) and used as received without further purification or treatment. First, PT $(18 \mathrm{mg})$ was dissolved in 1,2-dichlorobenzene (1,2-DCB) $(0.5 \mathrm{~mL})$ at a concentration of $36 \mathrm{mg} / \mathrm{mL}$. When the solution was heated, it was stirred and then cooled to room temperature, (R)$(+)$-limonene $(1 \mathrm{~mL})$ was added to the solution at a $2: 1$ volume ratio $((\mathrm{R})-(+)-$ limonene:1,2-DCB) to form a $12 \mathrm{mg} /$
$\mathrm{mL}$ mixed solution. Limonene, as a chiral inducer, plays a key role in the formation of chiral PT. With an increasing doping ratio of limonene, the $\mathrm{CD}$ signal becomes stronger and then becomes weaker. Thus, the chiral structure will change when tuning the limonene doping ratio. The optimal doping ratio is obtained and used in this work. Stirring the solution thoroughly induced the formation of a chiral structure. Finally, acetonitrile $(0.05 \mathrm{~mL})$ was added at a $10: 1$ volume ratio (1,2-DCB:ACN), and the solution was stirred to be fully mixed. After a period of standing, a chiral PT nanowire was formed. PCBM $(18 \mathrm{mg})$ was dissolved in the chiral PT solution $(1.5 \mathrm{~mL})$ at a $1: 1$ mass ratio to make the organic chiral charge transfer complexes. The PT:PCBM films are prepared by coating the mixed solution on glass to perform some characterization tests, including atomic force microscopy (AFM), photoluminescence spectroscopy, and CD.

\section{Device structure}

The ITO substrate was cleaned, and then chiral PT: PCBM solution was coated at $800 \mathrm{rpm}$ for $60 \mathrm{s.} \mathrm{MoO}_{3}$ and $\mathrm{Ag}$ were evaporated to the active layer in an orderly manner to make the device, which could be used to perform electric field-related tests, such as those for electric field-related PL and the dielectric constant.

\section{Measurements}

A JASCO J-810 meter with a response time of $1 \mathrm{~s}$ was used to characterize the CD spectra of the chiral PT films and chiral PT:PCBM films. PL was measured with a PG2000 Pro spectrometer, and the polarized state of light was analyzed through a polarimeter (PAX1000, Thorlabs) combined with quarter-wave plates and linear polarizers. The temperature dependence of spin polarization was characterized through a vibrating sample magnetometer (Lakeshore 8604). The dielectric constant measurements were performed with Agilent E4980A impedance instrument with a frequency 
range of $20 \mathrm{~Hz}$ to $2 \mathrm{MHz}$. The J-V curve of the device was characterized using a Keithley 2400 instrument (Keithley Instruments Inc., Cleveland, OH, USA). The thickness of the dielectric layer was measured with AFM.

\section{Acknowledgements}

This work was supported by the NSFC (Grant Nos. 91963103 and 62174102), Taishan Scholar Foundation of Shandong Province (tsqn201812007), Shandong Province Outstanding Youth Foundation (ZR2020JQ02), Major Program of Shandong Province Natural Science Foundation (ZR2019ZD43), and Shandong University Multidisciplinary Research and Innovation Team of Young Scholars (2020QNQT013).

\section{Author contributions}

W.Q. conceived the original idea. R.H. designed the experiments and performed the measurements. R.H. carried out the data analyses under the supervision of W.Q. Finally, R.H. and W.Q. wrote the paper. All authors discussed the results and commented on the manuscript.

\section{Conflict of interest}

The authors declare no competing interests.

\section{Publisher's note}

Springer Nature remains neutral with regard to jurisdictional claims in published maps and institutional affiliations.

Supplementary information The online version contains supplementary material available at https://doi.org/10.1038/s41427-022-00358-8.

Received: 18 October 2021 Revised: 21 December 2021 Accepted: 13 January 2022.

Published online: 4 February 2022

\section{References}

1. Young, H. K., Zhai, Y. \& Lu, H. Chiral-induced spin selectivity enables a roomtemperature spin light-emitting diode. Science 371, 1129-1131 (2021).

2. Chen, C. et al. Metasurfaces with planar chiral meta-atoms for spin light manipulation. Nano Lett. 21, 1815-1821 (2021).

3. De, A., Bhowmick, T. K. \& Lake, R. K. Anomalous magneto-optical effects in an antiferromagnet-topological-insulator heterostructure. Phys. Rev. Appl. 16, 014043 (2021).

4. Gu, B., Takahashi, S. \& Maekawa, S. Enhanced magneto-optical Kerr effect at Fe/insulator interfaces. Phys. Rev. B 96, 21 (2017).

5. Müller, T.-O. \& Müller, C. Longitudinal spin polarization in multiphoton BetheHeitler pair production. Phys. Rev. A 86, $86 \mathrm{~b} 2109$ (2021).

6. Zhou, X., Feng, W., Yang, X., Guo, G.Y. \& Yao, Y. Crystal chirality magnetooptical effects in collinear antiferromagnets. Phys. Rev. B 104, 024401 (2021).

7. Fang, Y., Wu, S., Zhu, Z.-Z. \& Guo, G.-Y. Large magneto-optical effects and magnetic anisotropy energy in two-dimensional Cr2Ge2Te6. Phys. Rev. B 98, 125416 (2021).

8. Abendroth, J. M. et al. Helicity-preserving metasurfaces for magnetooptical enhancement in ferromagnetic $[\mathrm{Pt} / \mathrm{Co}] \mathrm{N}$ films. Adv. Opt. Mater. 8, 2001420 (2020).

9. Stanciu, C. D. et al. All-optical magnetic recording with circularly polarized light. Phys. Rev. Lett. 99, 047601 (2021).

10. Boehme, C. \& Lupton, J. M. Challenges for organic spintronics. Nat. Nanotechnol. 8, 612-615 (2013).

11. Ghosh, R. \& Spano, F. C. Excitons and polarons in organic materials. Acc. Chem. Res. 53, 2201-2211 (2020).

12. Heeger, A. J., Kivelson, S., Schrieffer, J. R. \& Su, W. P. Solitons in conducting polymers. Rev. Mod. Phys. 60, 781-850 (1988).

13. Lv, X.-R., Liang, S.H., Tao, L.-L. \& Han, X.F. Organic spintronics: past, present and future. Spin 04, 02 (2014).
14. Rice, M. J., Phillpot, S. R., Bishop, A. R. \& Campbell, D. K. Solitons, polarons, and phonons in the infinite polyyne chain. Phys. Rev. B 34, 4139-4149 (1986).

15. Su, W. P., Schrieffer, J. R. \& Heeger, A. J. Solitons in polyacetylene. Phys. Rev. Lett. 42, 1698-1701 (1979).

16. Tarafder, K., Sanyal, B. \& Oppeneer, P. M. Charge-induced spin polarization in nonmagnetic organic moleculeAlq3. Phys. Rev. B 82, 060413 (2010).

17. Wei, M. et al. Organic multiferroic magnetoelastic complexes. Adv. Mater. 32 , 2003293 (2020).

18. Wang, Z. et al. Organic chiral charge transfer magnets. ACS Nano 13 4705-4711 (2019).

19. Wei, M., Fan, Y. \& Qin, W. Progress of organic magnetic materials. Sci. China Phys. Mech. Astron. 62, 977501 (2019).

20. Wang, Z. \& Qin, W. Organic magnetoelectric and optomagnetic couplings: perspectives for organic spin optoelectronics. NPG Asia Mater. 13, 17 (2021).

21. Basko, D. M. \& Conwell, E. M. Stationary polaron motion in a polymer chain at high electric fields. Phys. Rev. Lett. 88, 056401 (2002).

22. Jaeger, H. M., Fischer, S. \& Prezhdo, O. V. Decoherence-induced surface hopping. J. Chem. Phys. 137, 22 A545 (2012).

23. Johansson, A. A. \& Stafström, S. Nonadiabatic simulations of polaron dynamics. Phys. Rev. B 69, 235205 (2004).

24. Liu, X. et al. Effect of the electric field mode on the dynamic process of a polaron. Phys. Rev. B 74, 172301 (2006).

25. Mizes, H. A. \& Conwell, E. M. Stability of polarons in conducting polymers. Phys. Rev. Lett. 70, 1505-1508 (1993).

26. Johansson, A. \& Stafstrom, S. Polaron dynamics in a system of coupled conjugated polymer chains. Phys. Rev. Lett. 86, 3602-3605 (2001).

27. Liu, W., Gao, K., Li, Y., Sun, Z. \& Liu, D. Dynamics of interchain delocalized polarons in polymers. Sci. China Phys. Mech. Astron. 53, 315-320 (2010).

28. Wohlgenannt, M., Jiang, X. M. \& Vardeny, Z. V. Confined and delocalized polarons in $\pi$-conjugated oligomers and polymers: a study of the effective conjugation length. Phys. Rev. B 69, 24 (2004).

29. Yu, Z. G. Spin-orbit coupling and its effects in organic solids. Phys. Rev. B 85, 11 (2012).

30. Ron, N., Yossi, P. \& David, H. W. Chiral molecules and the electron spin. Nat. Rev. Chem. 3, 250-260 (2019).

31. John, M. A. et al. Spin selectivity in photoinduced charge-transfer mediated by chiral molecules. ACS Nano 13, 4928-4946 (2019).

32. Oren, B., Noam, M., Shira, Y., Lech, T. \& Yossi, P. Local light-induced magnetization using nanodots and chiral molecules. Nano Lett. 14, 6042-6049 (2014).

33. John, M. A. et al. Analyzing spin selectivity in DNA-mediated charge transfer via fluorescence microscopy. ACS Nano 11, 7516-7526 (2017).

34. Prakash, C. et al. Photospintronics: magnetic field-controlled photoemission and light-controlled spin transport in hybrid chiral oligopeptide-nanoparticle structures. Nano Lett. 16, 2806-2811 (2016).

35. Idé, J., Fazzi, D., Casalegno, M., Meille, S. V. \& Raos, G. Electron transport in crystalline PCBM-like fullerene derivatives: a comparative computational study. J. Mater. Chem. C 2, 7313-7325 (2014).

36. Smith, S. L. \& Chin, A. W. Phonon-assisted ultrafast charge separation in the PCBM band structure. Phys. Rev. B 91, 201302 (2015).

37. Harris, J. et al. Structured quantum waves. Nat. Phys. 11, 629-634 (2015).

38. Saitoh, K., Hasegawa, Y., Hirakawa, K., Tanaka, N. \& Uchida, M. Measuring the orbital angular momentum of electron vortex beams using a forked grating. Phys. Rev. Lett. 111, 074801 (2013).

39. Chico, L., Lopez-Sancho, M. P. \& Munoz, M. C. Spin splitting induced by spinorbit interaction in chiral nanotubes. Phys. Rev. Lett. 93, 176402 (2004).

40. Liu, S. Y. F., Sun, Y. \& Ko, C. M. Spin polarizations in a covariant angularmomentum-conserved chiral transport model. Phys. Rev. Lett. 125, 062301 (2020).

41. Hou, D., Qiu, J., Xie, S. \& Saxena, A. Charge-induced spin polarization in thiophene oligomers. N. J. Phys. 15, 1367-2630 (2013).

42. Köhler, A. \& Beljonne, D. The singlet-triplet exchange energy in conjugated polymers. Adv. Funct. Mater. 14, 11-18 (2004).

43. Veldman, D. et al. Compositional and electric field dependence of the dissociation of charge transfer excitons in alternating polyfluorene copolymer/ fullerene blends. J. Am. Chem. Soc. 130, 7721-7735 (2008).

44. Hanna, T. F., Johanna, D., Shira, Y., Efrat, L. \& Yossi, P. Spin-exciton delocalization enhancement in multilayer chiral linker/quantum dot structures. J. Phys. Chem. Lett. 10, 3858-3862 (2019). 